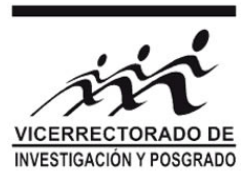

\title{
Implementación del Diplomado en Gestión Integrada de Recursos de Agua en el Perú
}

\author{
Vinio Floris ${ }^{*}$, Armando Gallegos ${ }^{1}$ y Teresa Velásquez ${ }^{2}$ \\ ${ }^{1}$ Programa de Gestión de Agua en Energía y Minería, GERENS, Perú \\ ${ }^{2}$ Universidad Nacional Agraria La Molina, Perú
}

Recibido 05 agosto 2019 - Aceptado 10 noviembre 2019

\begin{abstract}
Resumen
El consorcio liderado por la Escuela de Postgrado GERĚNS (Perú) y sus socios Colorado State University (EE. UU.) y la Fundación Chile (Chile), diseñaron e implementaron en Perú en el bienio 2014-2015 el Diplomado en Gestión Integrada de los Recursos Hídricos para Profesionales de Gerencia Media. Éste se ofreció a un centenar de profesionales de instituciones regionales y locales que conforman el Sistema Nacional de Gestión de los Recursos Hídricos como parte de la ejecución del Proyecto de Modernización de la Gestión de los Recursos Hídricos de la Autoridad Nacional del Agua (ANA). El diplomado produjo un impacto importante en los participantes y sus organizaciones, a juzgar por las expresiones positivas de los actores involucrados: alumnos, organizaciones patrocinadoras y los profesores mismos. Se observó mejoras en la capacidad de toma de decisiones, un avance en la gestión holística del recurso agua y el fortalecimiento de la carrera profesional de los participantes.

Palabras clave: Gestión Integral de los Recursos Hídricos, desarrollo de competencias.
\end{abstract}

\section{Implementation of the Diplomat in Integrated Management of Water Resources in Peru}

\begin{abstract}
The Consortium led by GERĚNS Postgraduate School (Peru) and its partners, Colorado State University (USA) and Fundación Chile (Chile), designed and implemented a Graduate Diploma Program on Integrated Water Resources Management for Mid-career professionals in Peru during 2014-2015. It was offered to over a hundred regional and local professionals from several institutions that belong to the National Water Resources Management System as part of the Modernizationof the Water Resources Management Program of the National Water Resources Authority (ANA). The Graduate Diploma Program had a significant impact on its participants and organizations based on the positive comments of the actors involved: students, sponsoring organizations and instructors. Considerable improvements have been observed in the decisionmaking-process, progress in the holistic approach of water resources management, and a bolstering of the professional careers of the participants.
\end{abstract}

Keywords: Integral Management of Water Resources, skills development.

\section{Introducción}

En el Perú, la normativa del agua ordena los roles y competencias de los distintos actores que intervienen en su gestión. Este nuevo enfoque aporta una visión multisectorial a la gestión de los recursos hídricos, consignando las cuencas como unidades hidrográficas (aguas superficiales) y acuíferas (aguas subterráneas) [lnb09]. Además promueve explícitamente el uso eficiente y el ahorro de agua, incluyendo la aplicación de incentivos. La entidad responsable y ejecutora de este marco es la Autoridad Nacional de Agua (ANA) [Ana13] y su Sistema Nacional de la Gestión de los Recursos Hídricos (SNGRH).

Una de las prioridades de la ANA para alcanzar sus objetivos es el desarrollar las competencias técnicas y las capacidades de gestión de sus miembros ejecutores. El consorcio liderado por GERĚNS Escuela de Postgrado y sus socios Colorado State University y la Fundación Chile; ganó el concurso internacional convocado por la ANA y durante los años 2014 y 2015 diseñó e implementó el 
Diplomado de Gestión Integrada de los Recursos Hídricos para Profesionales de Gerencia Media. El objetivo del programa fue el fortalecimiento institucional con una atención especial en la gestión integrada de recursos hídricos, el establecimiento de una cultura de gestión del agua y el desarrollo de capacidades para la gestión del agua en el nivel intermedio, incluyendo el personal de la ANA a nivel local y regional y el personal que se encuentra trabajando en el Sistema Nacional de Gestión de Recursos Hídricos [Cim12], [Cim15].

El diplomado consistió en un programa académico que contiene una secuencia de clases teóricoprácticas, interacción de estudiantes y profesores a través de un portal electrónico https://gerens.pe/ educacion-ejecutiva/, monitoreo y "coaching" (con mentores previamente asignados), visitas de campo, un trabajo final (grupal) aplicativo de mejora, pasantías en Colorado State University (Fort Collins, Colorado, Estados Unidos de América) y la Fundación Chile (Santiago, Chile) y una rigurosa evaluación de los estudiantes con vigilancia de su rendimiento en todas las etapas del programa. El mismo estuvo dirigido a fortalecer las capacidades de gestión de los profesionales de la gerencia media de la ANA, las instituciones regionales y locales que forman parte del SNGRH con el fin de que puedan contribuir a la creación de valor a través de la toma de decisiones concertadas en el uso óptimo del recurso agua, considerando los aspectos técnicos, económicos, sociales y ambientales, en el marco del desarrollo sostenible [|lfc12]. Este programa se desarrolló en estrecha coordinación entre el Proyecto de
Modernización de la Gestión de los Recursos Hídricos y la Dirección de Gestión del Conocimiento y Coordinación Interinstitucional de la ANA con el financiamiento del Banco Mundial [Gri16].

El diplomado se ofreció para 103 profesionales de instituciones regionales y locales (públicas, privadas y no gubernamentales) que forman parte del SNGRH. Este evento se realizó en tres en regiones del país con sedes en Chiclayo (norte), Lima (centro) y Arequipa (sur).

El diplomado produjo un impacto importante en los participantes y sus organizaciones, a juzgar por las expresiones positivas de los actores involucrados: alumnos, organizaciones patrocinadoras y los profesores mismos. Se observómejoras en la capacidad de toma de decisiones, un avance en la gestión holística del recurso agua y el fortalecimiento de la carrera profesional de los participantes.

\section{Objetivos}

Este artículo busca poner al alcance de la comunidad académica, gubernamental y empresarial los aprendizajes logrados con una experiencia pionera e innovadora en la formación de talento humano requerido para la gestión integrada de recursos hídricos. Los objetivos específicos son los siguientes:

a. Presentar el diseño del diplomado, sus cursos y selección de instructores y tutores.

b. Discutir la organización e implementación del diplomado.

c. Reflexionar sobre los resultados, contribuciones y lecciones aprendidas.

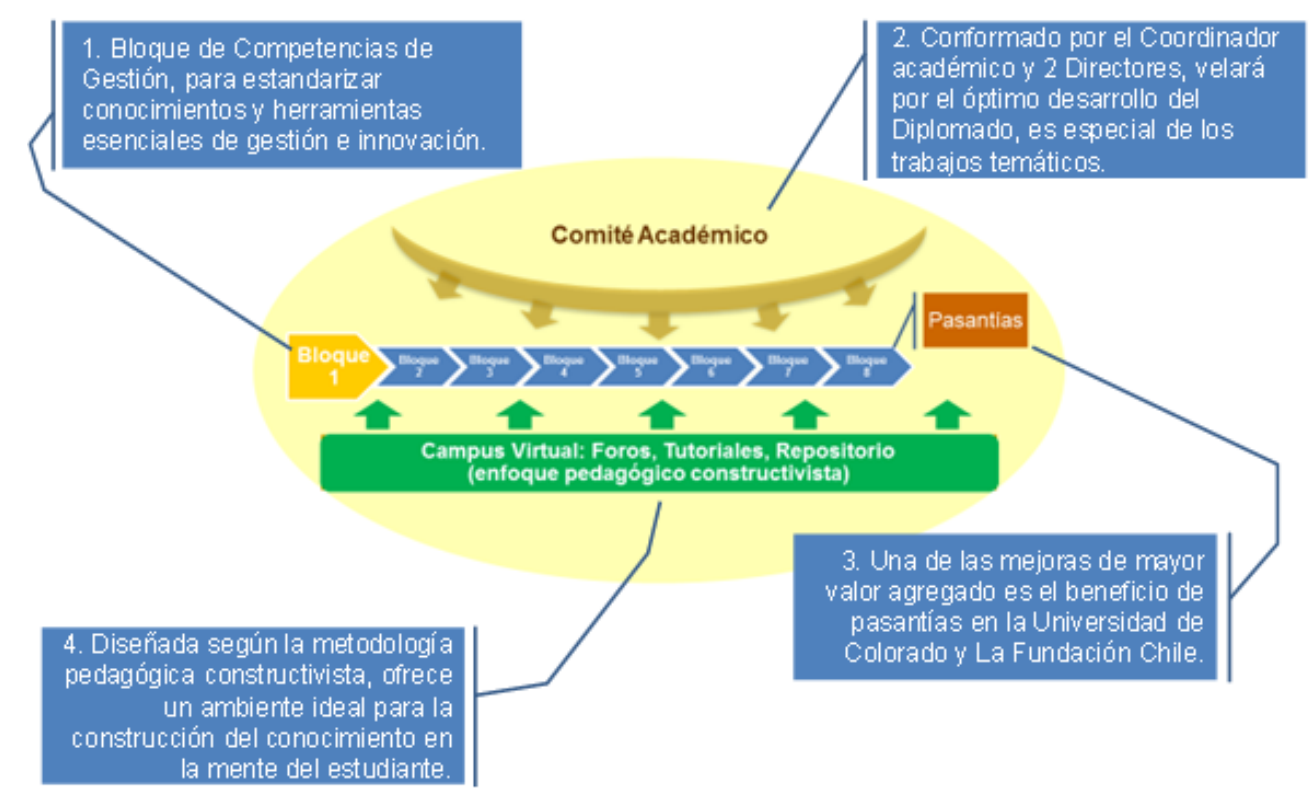

Figura 1: Esquema del diseño del Diplomado de Gestión Integrada de Recursos de Agua. 


\section{Metodología}

\section{Diseño de la malla curricular}

El diplomado fue diseñado por un selecto grupo de profesores y expertos que mantuvieron un estrecho dialogo con la ANA. Todo este programa tuvo ocho bloques (más uno adicional - el Bloque 1- para la organización del evento) y las respectivas pasantías. Se constituyó un Comité Académico (compuesto por un coordinador académico y dos directores) que constantemente supervisaron el desarrollo de éste. Se dieron un total de 270 horas lectivas (Figura 1).

\section{Implementación}

La implementación se inició con un proceso de selección de candidatos de las tres regiones que fueron sedes. Se eligieron y graduaron 103 participantes. Los estudiantes fueron rigurosamente evaluados; aquellos más destacados fueron becados con pasantías en Estados Unidos de América (con Colorado State University) y en Chile (con la Fundación Chile). La Figura 2 muestra los contenidos de los ocho bloques.

El diplomado fue ejecutado bajo un enfoque de pedagogía constructivista que se mueve de un escenario de un profesor experto, currículo vertical de conocimiento estático y alumno pasivo a uno de profesor mediador (sin dejar de ser experto), currículo horizontal (flexible y modificable) de conocimiento activo y alumno constructor de su propio aprendizaje [Gra19].

Los profesores y directores de bloque también fueron seleccionados rigurosamente, todos ellos con doctorados y/o maestrías en universidades de primer nivel y gran ex- periencia en enseñanza y/o gestión de recursos de agua. El diplomado tuvo un total de 18 profesores y tutores de diversas nacionalidades y regiones de las Américas.

El diplomado estuvo basado en clases teórico-prácticas (con sílabos especialmente desarrollados), estudios de casos y experiencias prácticas de gestión integral de recursos hídricos en Perú y otros países, análisis de documentos y fuentes secundarias y visitas de campo. Las presentaciones, notas de clase y casos siguieron el enfoque andragógico (materiales didácticos enfocados al público adulto y experimentado) con considerable participación de los asistentes. La enseñanza fue apalancada con el uso de la Plataforma Virtual MOODLE (conocida como Learning Content Management System), una aplicación web de tipo Ambiente Educativo Virtual que ofrece un sistema completo de gestión de cursos y ayuda a los profesores a crear comunidades de aprendizaje en línea. Todos los participantes tuvieron que completar el Trabajo Aplicativo de Mejora (TAM), una tesina grupal que demostró lo aprendido en todo el evento. Las pautas, responsabilidades y obligaciones de los participantes y de los docentes se especificaron en un reglamento general que ayudó a la planificación ejecución y control del programa.

\section{Secuencia y contenido de los bloques}

El contenido de los ocho bloques lectivos (cuatro de gestión de recursos hídricos, tres de ingeniería de recursos de agua y uno de especialización), sus respectivos módulos (uno o dos, dependiendo del caso), talleres, visitas al campo y los exámenes finales. Los ocho bloques fueron clasificados en dos ciclos (I y II) los cuales son mostrados en las Figuras 3 y 4 respectivamente.

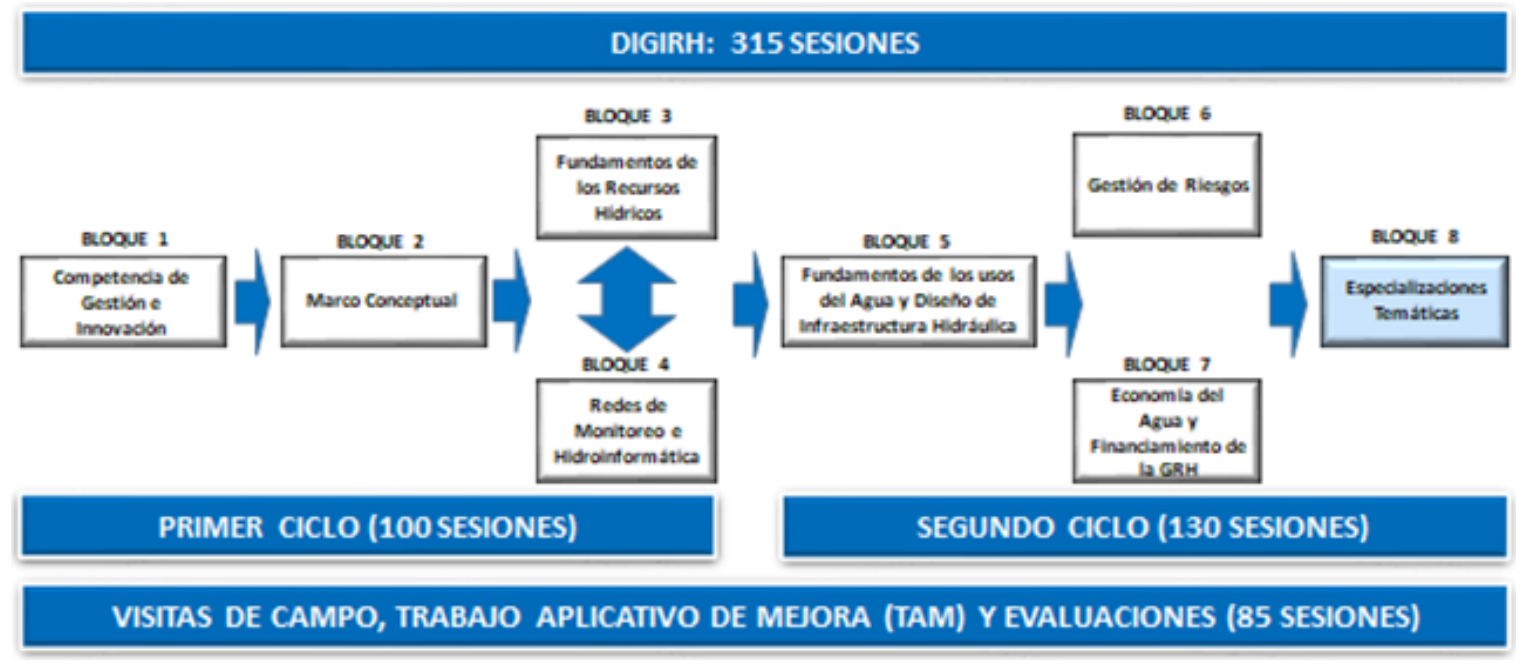

Figura 2: Estructura del Diplomado de Gestión Integrada de Recursos de Agua. 


\section{Metodología Constructivista}

El programa se desarrolló empleando el método de aprendizaje constructivista el cual propugna que el proceso enseñanza-aprendizaje está centrado en el participante. Es este mismo el que construye su propio conocimiento desarrollando su capacidad de investigar, pensar, reflexionar y adquirir experiencias que le permiten acceder a sistemas más complejos, propios de niveles superiores. El rol del docente es facilitar el proceso creando un ambiente adecuado y motivando a los participantes a acceder a estructuras cognitivas de la etapa inmediatamente superior (Figura 5).

En el caso de los Cursos, se incluyeron la exposición de estudios de casos y se logró la participación de diversas opiniones de experiencias similares en el Perú y extranjero. Así mismo se desarrolló el TAM con temas actuales y presentes seleccionados por los mismos participantes, los que se desarrollaron en reuniones específicamente diseñadas para tal fin. El avance de temas fue guiado a través de formatos con pautas e hitos de los alcances, que fueron presentados por los grupos de trabajo en cada una de las sesiones diseñadas.

\begin{tabular}{|c|c|c|c|}
\hline & Bloques & Módulos & $\begin{array}{c}\text { Horas } \\
\text { académicas } \\
\left(45^{\circ}\right)\end{array}$ \\
\hline \multirow{9}{*}{$\underset{1}{\mathrm{CICLO}}$} & \multirow{2}{*}{$\begin{array}{l}\text { Bloque 1: } \\
\text { Competencias } \\
\text { de gestión e } \\
\text { innovación }\end{array}$} & Módulo I: Desarrollo de competencias de gestión & \multirow{2}{*}{30} \\
\hline & & $\begin{array}{l}\text { Módulo II: Soluciones innovadoras para la gestión del } \\
\text { agua }\end{array}$ & \\
\hline & $\begin{array}{l}\text { Bloque 2: } \\
\text { Marco } \\
\text { conceptual }\end{array}$ & $\begin{array}{l}\text { Módulo I: Bases para la gestión integrada de los } \\
\text { recursos hidricos (GIRH) }\end{array}$ & 15 \\
\hline & \multirow{2}{*}{$\begin{array}{l}\text { Bloque 3: } \\
\text { Fundamentos } \\
\text { de los recursos } \\
\text { hidricos }\end{array}$} & Módulo I: Hidrologia y cambio climático & \multirow{2}{*}{26} \\
\hline & & Módulo II: Saneamiento ambiental y calidad del agua & \\
\hline & \multirow{2}{*}{$\begin{array}{l}\text { Bloque 4: } \\
\text { Redes de } \\
\text { monitoreo e } \\
\text { hidroinformática }\end{array}$} & Módulo I: Hidrometria & \multirow{2}{*}{30} \\
\hline & & Módulo II: Informática aplicada a los recursos hidricos & \\
\hline & \multicolumn{2}{|c|}{$\begin{array}{l}\text { TALLERES - Alineamiento de bloques del ciclo } 1 \text { con el Trabajo } \\
\text { Aplicativo de Mejora }\end{array}$} & 19 \\
\hline & \multicolumn{2}{|c|}{ VISITA DE CAMPO } & 19 \\
\hline
\end{tabular}

Figura 3: Secuencia y contenido de los Bloques 1 a 4.

El diplomado fue sustentado por un sistema de soporte que actuó como una plataforma virtual a fin de facilitar la interacción entre alumnos, profesores y tutores. La plataforma fue el medio de intercambio de opiniones e información la cual buscó facilitarla participación e intercambio de aportes y experiencias.

\section{Beneficios de los participantes}

Los beneficios de los participantes fueron:

- Involucramiento directo y aprendizaje de una visión de un modelo integral, herramientas y metodologías prác- ticas vinculadas con aspectos claves en la gestión de los recursos hídricos.

- Compartir esta experiencia académica teóricopráctica con un equipo de profesores que forman parte de tres instituciones líderes en educación e investigación en la gestión de recursos hídricos: la Escuela de Postgrado GERENS, la Colorado State University y la Fundación Chile.

- Análisis práctico de la gestión de los recursos hídricos a través del desarrollo de un TAM como trabajo final y requisito de graduación.

- Pasantías en Colorado State University y la Fundación Chile para los participantes que ocupen los primeros puestos inmediatamente después de concluido el diplomado.

\section{Instructores y mentores}

El diplomado tuvo 18 docentes de primer nivel, 9 peruanos y 9 procedentes de Chile, México y Estados Unidos; todos con doctorados y/o maestrías en Universidades de primer nivel, como: Colorado State University, Universidad Nacional Mayor de San Marcos, Universidad Nacional Agraria la Molina, University of Texas-Austin, Harvard University, Utah State University, y con amplia experiencia académica y gerencial. Algunos de estos profesionales fungieron como mentores pero en su mayoría fueron otros expertos que se dedicaron exclusivamente a la mentoría.

\section{Resultados}

Basada en la evaluación del diplomado y el seguimiento de las actividades de sus participantes, se concluyó que los resultados del programa de desarrollo de competencias excedieron las expectativas planteadas. He aquí un resumen de éstas:

a. Diseño integrado de un programa multidisciplinario. El enfoque constructivista hizo que los participantes, con más de 20 profesiones diversas y decenas de títulos y obligaciones laborales, pudieran integrarse y hacer uso, en conjunto, de los principios y técnicas de gestión del recurso hídrico.

b. Integración de temas técnicos y de gestión. Fue retador para el diseño académico lograr que los módulos aborden simultáneamente, por un lado temas especializados y técnicos referidos a la gestión del agua, y por otro los aspectos de gestión integrada del recurso hídrico, que la toma de decisiones y la consideración de aspectos financieros, legales, socio-políticos, ambientales, e interacción, influencia y negociación con grupos de interés (o “stakeholders") [Cmr00], [Cfi12.

c. Nivel de aprendizaje. La gran mayoría obtuvo altas calificaciones de aprobación (promedio de B+ y A-). En discusiones informales con los alumnos, muchos se sienten mucho más preparados para enfrentar no sólo sus tareas 
cotidianas sino también en asumir nuevos retos profesionales.

\begin{tabular}{|c|c|c|c|}
\hline & Bloques & Módulos & $\begin{array}{c}\text { Horas } \\
\text { académicas } \\
\left(45^{\prime}\right)\end{array}$ \\
\hline \multirow{9}{*}{$\begin{array}{c}\text { CICLO } \\
2\end{array}$} & \multirow{2}{*}{$\begin{array}{l}\text { Bloque 5: } \\
\text { Fundamentos } \\
\text { de los usos del } \\
\text { agua y diseño } \\
\text { de } \\
\text { infraestructura } \\
\text { hidráulica }\end{array}$} & Módulo I: Infraestructura en los recursos hidricos & \multirow[b]{2}{*}{30} \\
\hline & & Módulo II: Marco legal de los recursos hidricos & \\
\hline & \multirow{2}{*}{$\begin{array}{l}\text { Bloque 6: } \\
\text { Gestión de } \\
\text { riesgos }\end{array}$} & Módulo I: Gestión y análisis de inundaciones & \multirow{2}{*}{27} \\
\hline & & Módulo II: Gobernanza, gestión y adaptación & \\
\hline & \multirow{2}{*}{$\begin{array}{l}\text { Bloque 7: } \\
\text { Economia del } \\
\text { agua y } \\
\text { financiamiento } \\
\text { de la gestión de } \\
\text { recursos } \\
\text { hidricos }\end{array}$} & Módulo I: Análisis económico y financiero & \multirow{2}{*}{30} \\
\hline & & Módulo II: Economia del agua & \\
\hline & $\begin{array}{l}\text { Bloque 8: } \\
\text { Especialidades } \\
\text { temáticas } \\
\text { (optativas) }\end{array}$ & $\begin{array}{l}\text { Los participantes podrán optar por desarrollar } \\
\text { diferentes módulos que abordarán temas } \\
\text { especializados y relevantes para la gestión de los } \\
\text { recursos hidricos. Las opciones de módulos / temas se } \\
\text { anunciarán al iniciar el ciclo } 2 \text {. }\end{array}$ & 45 \\
\hline & \multicolumn{2}{|c|}{$\begin{array}{l}\text { TALLERES - Alineamiento de bloques del ciclo } 1 \text { con el Trabajo } \\
\text { Aplicativo de Mejora }\end{array}$} & 18 \\
\hline & \multicolumn{2}{|c|}{ VISITA DE CAMPO } & 10 \\
\hline
\end{tabular}

EXÁMENES - evaluación de los bloques desarrollados en cada ciclo EVALUACIÓN FINAL DEL. TRABAJO APLICATIVO DE MEJORA

Figura 4: Secuencia y contenido de los Bloques 5 á 8 .

d. Trabajo Aplicativo de Mejora (TAM). Contribuyó altamente al aprendizaje y a la solución de retos de gestión en áreas específicas de interés. Por ejemplo, los estudiantes de la sede de Lima mostraron tendencias en la planificación de recursos hídricos. Los de la sede de Chiclayo (norte del Perú) se orientaron en temas relacionados a la administración del agua (por ejemplo, eficiencias, tarifas, cobros y moras) y resolver problemas propios de la región. Los de la sede de Arequipa (sur del Perú) se encaminaron a resolver temas críticos con soluciones innovadoras con uso de considerables experiencias propias de los participantes. Once de los trabajos fueron recomendados por el Comité Académico a la ANA para ser publicados como estudios de caso y/o material de estudio por su alta calidad.

e. Barrera de la distancia física. La plataforma Virtual MOODLE cumplió su cometido de constituirse en un repositorio de todo el material del diplomado y eliminando las distancias geográficas de los participantes (alumnos viajaban horas y hasta días para tomar las clases en sus sedes; muchos profesores tienen residencia en el extranjero a miles de kilómetros de distancia). Sin embargo, no se logró la plena utilización de las capacidades de la plataforma principalmente por temas de cultura informática y hábitos tanto de los alumnos como de los profesores. A futuro debe abordarse este reto.

f. Seguimiento. GERĚNS aspira a hacer un seguimiento de los egresados a fin de comprobar la eficacia del apren- dizaje logrado. Una lección aprendida es la necesidad de crear un sistema posgraduación que provea retroalimentación sobre el impacto de la capacitación dada y sea a la vez un sistema de educación suplementaria continua.

\section{Conclusiones}

Las conclusiones sobre las lecciones aprendidas se pueden sintetizar en los siguientes:

a. El Consorcio sumó capacidades organizacionales. El consorcio produjo importantes sinergias pues sumó fortalezas de las tres instituciones que aportaron expertos con profundidad de conocimiento y experiencia en las disciplinas relevantes. Se constituyó una base de conocimientos en que se cimentó el diplomado y llevó a desarrollar contenidos coherentes y relevantes, diseñar un plan de actividades altamente efectivo y una organización que se constituyó en una gran fortaleza.

b. Atributos diferenciadores del programa. El consorcio incorporó en el diseño del diplomado varios atributos que tuvieron un gran impacto positivo en el programa: módulo de gestión, pasantías para los alumnos más destacados en Colorado State University y la Fundación de Chile, un plantel de profesores con gran experiencia y habilidades (57\% con Ph.D. y $43 \%$ con maestrías) y con un Comité Académico eficiente. Estos atributos probaron ser sumamente valiosos y diferenciadores para el programa.

c. Organización académica adecuada. El diplomado tuvo una estructura organizacional que probó ser altamente efectiva. El liderazgo de la gestión académica estuvo a cargo del Comité Académico y la coordinación académica del programa. Esta instancia jugó un rol vital en la adecuación de la metodología y en el trabajo cercano de los profesores y directores de bloque en la preparación detallada y desarrollo de los cursos. Adicionalmente, fue clave el apoyo e interacción con los profesores en la estructuración y asignación de horas por temas ya que se clarificó la visión general del diplomado y los objetivos esperados con cada curso, diseño detallado de los sílabos y materiales de trabajo. Cada bloque tuvo un director con una amplia experiencia y conocimiento de éste y trabajó con losprofesores asignados y la coordinación académica en la implementación del bloque. La tutoríacumplió un rol clave al asesorar y apoyar cercanamente a los equipos de alumnos en la elaboración del TAM.

d. Competencias de gestión. Se incluyó dos bloques con un fuerte énfasis en competencias de gestión. El Bloque 1 tuvo como objetivo estandarizar conocimientos, habilidades y actitudes en gestión e innovación las cuales son áreas identificadas como deficitarias en la formación profesional en el Perú, con gran importancia en la gestión de recursos hídricos. El Bloque 8, que conformó los cursos electivos del diplomado se enfocó en fortalecer competencias de gestión. Incluyó módulos sobre métodos de análisis 
y solución de problemas a fin de mejorar su capacidad de implementación de proyectos y estrategias para la gestión integrada de los recursos hídricos; gestión de relaciones con los grupos de interés, gestión del agua bajo un enfoque holístico así como una estrategia y gestión de los recursos hídricos bajo un enfoque integrado.

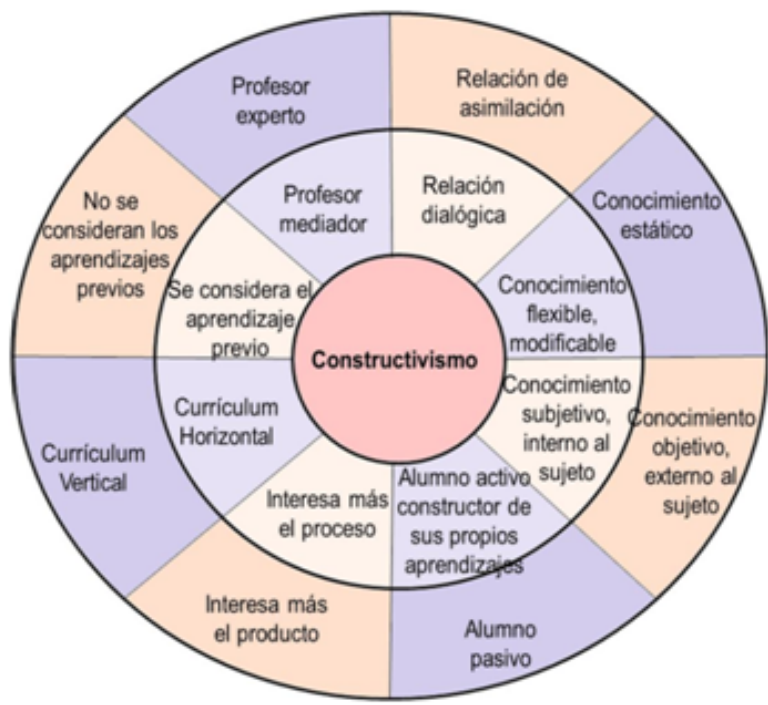

Figura 5: El enfoque de la pedagogía constructivista. Fuente: http://uoctic-grupo6.wikispaces.com/Constructivismo

e. Balance entre el contenido técnico y el contenido de gestión. Un constante reto en el diplomado fue el tratamiento balanceado de contenidos técnicos y los contenidos de gestión y toma de decisiones, al interior de cada bloque y cada módulo. Por ejemplo en el curso Fundamentos de los Usos del Agua, luego de varias aproximaciones sucesivas se transformó un sílabo con un enfoque técnico y con alto contenido matemático a uno orientado a la gestión y toma de decisiones en los aspectos fundamentales del uso del agua. Igualmente, en el curso de Hidráulica se buscó reducir la extensión de fórmulas y modelos matemáticos y enfocarse más en explicar casuística y los aspectos prácticos acerca de cómo utilizar esta materiaen la gestión integrada de los recursos hídricos. En los cursos de herramientas de gestión, como por ejemplo Formulación de Problemas Complejos o en Gestión de Relaciones con los Tomadores de Decisión, el reto fue inverso; se enfocó en cómo aplicar dichas herramientas en el contexto de laproblemática de los recursos hídricos. Ello se logró con la preparación de casos y aplicaciones a la gestión de cuencas, Autoridad Locales de Aguas y otras realidades organizacionales e institucionales relevantes. La adecuación en el diseño del curso para obtener el balance adecuado fue logrado con más éxito en unos cursos que en otros pero en suma se logró el objetivo, agregando los módulos 1 y 8.

f. Exigencia académica. El diplomado tuvo elevadas exigencias académicas que incluyeron exámenes individuales escritos por ciclo, trabajos aplicativos con notas grupales, visitas de campo con talleres de trabajo y la elaboración de un proyecto de investigación que fue sustentado ante un jurado. Adicionalmente los niveles de asistencia fueron altos.

g. Pedagogía constructivista. La aplicación de la pedagogía constructivista se constituyó en uno de los pilares del diplomado. Para lograrlos trabajaron en conjunto los profesores, la coordinación académica y el Comité Académico. Se buscó que cada módulo tenga impacto en las dimensiones actitudinales y que los sílabos se adapten a lo que plantea el enfoque constructivista. Se buscó el aprendizaje significativo adaptando los contenidos a la problemática institucional, socio-política y técnica de la gestión del agua; se buscó el aprendizaje centrado en la persona, con los trabajos aplicativos y tareas encargadas, coaching individualizado a los equipos y alumnos. Finalmente, se aplicó la metodología activa, planificando situaciones de aprendizaje estimulantes como la discusión de casos, las visitas de campo, y las pasantías que actuaron como un recurso que motivó fuertemente a los participantes. Como dificultad para el empleo del método constructivista, se encontró la capacitación previa de algunos profesores más habituados al método tradicional centrado en el profesorexperto llevó a unainteracción personalizada y a un apoyo por parte de la coordinación académica.

h. Plataforma virtual Moodle. Se empleó en la Plataforma MOODLE, una aplicación web que proporcionó un ambiente educativo virtual consistente en un sistema completo de gestión de cursos, que ayudar a los educadores a crear comunidades de aprendizaje en línea.

i. La plataforma tuvo un repositorio de todos los materiales relevantes del diplomado: presentaciones, lecturas, foros. Adicionalmente se abrieron secciones para los trabajos aplicativos y la monografía; se buscó extender el uso de la plataforma para mejorar la interacción entre participantes, profesores, tutores y coordinación académica en las tres sedes. El uso pleno de este recurso más allá de ser un repositorio estuvo limitado por la baja inclinación de la mayoría de los alumnos y de algunos profesores a emplear más proactivamente el campus virtual y también debido a problemas de accesibilidad desde lugares remotos que tuvieron algunos participantes. La plataforma es una poderosa herramienta que amplifica las posibilidades de enseñanza aprendizaje. Esta fue sin duda una de las lecciones aprendidas más relevantes.

j. Tesina grupal. Uno de los requisitos académicos del Diplomado fue el desarrollo de una investigación realizada en equipo con el apoyo de un tutor y la coordinadora académica. Los TAM fueron sustentados ante un jurado. El resultado mostros un buen nivel técnico y el Comité Académico recomendó la publicación del $50 \%$ de los trabajos que cumplieron los siguientes criterios: (i) innovación, (ii) replicabilidad, (iii) enfoque GIRH, (iv) consideración de 
Tomadores de decisión, y (v) pertinencia y solidez de la metodología aplicada.Se identificó los siguientes factores como decisivos para el éxito de las monografías: (i) elección del tema por la cual se propuso un "tema sombrilla" para la monografía; es decir, que permita la aplicación holística de varias de las herramientas/conceptos del curso; (ii) se programó talleres de trabajo de día completo para que los equipos avancen y expongan dichos avances en aula, con la tutoría del caso; (iii) se proporcionó plantillas y formatos semiestructurados para facilitar el desarrollo de las monografías y ayudar a los alumnos a enfocarse en el fondo y dejar la forma en un segundo plano; y (iv) se programaron exposiciones de avances de la monografía al final del primer trimestre y al final del segundo trimestre. Se expuso la monografía completa al final de tercer trimestre, ante un jurado como cierre del Diplomado. Todo el proceso siguió un reglamento de tesina previamente desarrollado y divulgado.

k. Requisito de participación y tasa de deserción. Los participantes del Diplomado recibieron una beca de parte de la ANA. Uno de los logros del programa fue la baja tasa de deserción de los alumnos, la cual fue menor al $5 \%$. Esto se logró gracias a una combinación de motivación a los participantes y una gestión exigente pero flexible para el cumplimiento de los requisitos de puntualidad y asistencia. Se admitió faltas justificadas las cuales debían ser compensadas por un trabajo asignado al participante para recuperar la materia tratada en clase (además de cumplir con todos los trabajos asignados en la fecha). Sin embargo, se observó la falta de políticas complementarias de gestión de recursos humanos en retención y línea de carrera para los funcionarios capacitados así como descoordinaciones de la institución patrocinadora asignando al participante viajes o actividades y reuniones que se interponían con las clases.

I. Visitas de campo. Se hicieron dos visitas de campo en cada sede a fin de observar in situ los problemas de gestión existentes. Las mismas fueron eficaces vehículos de aprendizaje. Previamente la Coordinación Académica visitó la zona, contactó a los representantes de las instituciones y empresas y se estructuró la visita. Durante la misma se programaron actividades de discusión y una reunión plenaria con las instituciones del lugar y los actores claves. Los alumnos presentaron trabajos individuales y recibieron una evaluación por los mismos. Las visitas incidieron en temas tales como gestión integrada multisectorial y gestión de riesgos asociados al Fenómeno de El Niño.

\section{Referencias}

[Cim12] Consejo Internacional de Minería y Metales (ICMM). (2012). Water management in mining. A selection of case Studies.

[Cim15] Consejo Internacional de Minería y Metales (ICMM). (2015). Una Guía práctica para la gestión del agua a nivel cuenca para la industria minera y metalúrgica.

[Cmr00] Comisión Mundial de Represas (2000). Represas y desarrollo. Un nuevo marco para la toma de decisiones.

[Cfi12] Corporación Financiera Internacional (IFC por sus siglas en inglés) (2012). Guías sobre medio a mbiente, salud y seguridad. Washington, D.C., EE. UU.

[Ifc12] Corporación Financiera Internacional (IFC por sus siglas en inglés) (2012). Normas de Desempeño so- bre Sostenibilidad Ambiental y Social. Washington, D.C., EE. UU.

[Gra19] Gray, A. (2019). Constructivist Teaching and Learning. Ubicado en la siguiente página web: http://www.saskschoolboards.ca/old/ ResearchAndDevelopment/ResearchReports / Instruction/97-07.htm

[Inb09] International Network of Basins Organizations, Global Water Partnership (2009). Manual para la Gestión Integrada de Recursos Hídricos en Cuencas.

[Ana13] Autoridad Nacional del Agua-ANA-PMGRH-BM (2013). Diagnóstico y Plan de Desarrollo de Capacidades en Cultura del Agua para Profesionales de Gerencia Media en el Ámbito Nacional y en las Cuencas. Consorcio Inclam-Alternativa. Lima, Perú.

[Gri16] Grigg, N. (2016) Diplomat in Integrated Water Resources Management Assessment of Program Quality. Colorado State University. 
